\title{
Sistema energéticamente eficiente y de bajo costo para controlar la temperatura y aumentar el oxígeno en estanques de cultivo de alevines de tilapia roja
}

\author{
An Energy Efficient and Low Cost Sustem, to Control the \\ Temperature and the Oxygen Increase, in the Red Tilapia \\ Fingerling Cultivation's Tanks
}

\section{Resumen}

El artículo expone un sistema automático para controlar la temperatura y aumentar el oxígeno en estanques de cultivo de alevines de tilapia roja en la granja John Jairo González, del Politécnico Colombiano Jaime Isaza Cadavid. El control se realiza a través de un PLC que recibe dos señales de temperatura ubicadas en puntos diferentes del estanque, y una señal de un sensor de nivel. Ejecutando un algoritmo de control, activa o desactiva diferentes circuitos de

\section{Abstract}

This paper describes an automated system which controls the temperature and increases the oxygen, in the growing red tilapia fingerling's tanks, at the Politécnico Colombiano Jaime Isaza Cadavid's John Jairo González Farm. The control operates through a PLC, which receives two temperature signals from the tank's different parts, and another signal from a level sensor. The PLC runs a control algorithm, which enables or disables various cold water circuits, pumped from the Guaracú stream

\footnotetext{
* M.Sc. Politécnico Colombiano Jaime Isaza Cadavid (Medellín-Antioquia, Colombia). rdvasquez@elpoli.edu.co

** Politécnico Colombiano Jaime Isaza Cadavid (Medellín-Antioquia, Colombia). acamilop@hotmail.com

*** Politécnico Colombiano Jaime Isaza Cadavid (Medellín-Antioquia, Colombia). tito_aguas@hotmail.com
} 
Sistema energéticamente eficiente y de bajo costo para controlar la temperatura y aumentar el oxígeno en estanques de cultivo de alevines de tilapia roja

agua fría, proveniente de la quebrada Guaracú, o caliente, a través de un proceso de bombeo de agua del estanque hacia los colectores solares, y regresándola al estanque, recirculación que se hace hasta obtener una temperatura de $29^{\circ} \mathrm{C}$, un poco por encima de la ideal de $28^{\circ} \mathrm{C}$. El sistema de control es validado comparando temperatura y oxígeno con un tanque sin control.

Palabras clave: Alevines, Cultivo de Tilapia Roja, Controlador Lógico Programable, Electroválvulas, Motobomba. or the hot water through a pumping process, from the tank into the solar collectors, and then returning it to the tank. This recycling ends when it reaches, a $29^{\circ} \mathrm{C}$ temperature, slightly above the $28{ }^{\circ} \mathrm{C}$ ideal. The control system is validated by comparing the temperature and the oxygen, with a tank without any control.

Keywords: Fingerlings, Red Tilapia Growing, Programmable Logic Controller, Electro Valve, Water Pump. 


\section{INTRODUCCIÓN}

La tilapia roja es considerada uno de los peces con mayor futuro comercial, debido a su corto período de crecimiento y a su alta adaptabilidad a los ambientes de producción a los que se enfrenta $[1,2]$. La importancia de la cadena de la tilapia radica en su representatividad para el agro en el país, cuya capacidad está representada por el aumento en sus niveles de producción, en la generación de empleo rural y en su potencial exportador, lo que la ha posicionado como una de las cadenas más promisorias.

Las propiedades de la tilapia, en particular su carne blanca, su fácil fileteado, las escasas espinas, el suave sabor y la versatilidad en la cocción, la ubican como uno de los peces más comercializados en el ámbito internacional. En el mercado estadounidense, por ejemplo, ha logrado establecerse como uno de los diez más consumidos [3].

Es notable que en Colombia, el sector agroindustrial en actividades de piscicultura no goza de técnicas modernas ni grandes inversiones en innovación tecnológica. En los criaderos de alevines es muy importante mantener los parámetros en rangos óptimos de operación, como lo son la temperatura y el nivel de oxígeno, para lograr producciones más eficientes; para mejorar estas necesidades es posible recurrir a nuevas técnicas de control.

Este artículo se estructura de la siguiente manera: en la unidad dos se explican los problemas del estanque para la adecuada reproducción de los peces, al igual que datos de interés para mejorar el sistema de control; en la unidad tres se explican los instrumentos por utilizar para el sistema de control y el funcionamiento de cada uno de ellos; la unidad cuatro presenta el proceso que se siguió para la implementación y puesta en marcha del sistema; en el capítulo cinco se da una explicación de los resultados obtenidos, específicamente, para las variables temperatura y nivel de oxígeno disuelto, y, por último, el capítulo seis presenta las conclusiones del proyecto.

\section{El CULTIVo de ALEVINES DE TILAPIA ROJA EN EL CENTRO EXPERIMENTAL}

El estanque de peces se alimenta de agua proveniente de la quebrada Guaracú, que tiene una temperatura promedio de $23{ }^{\circ} \mathrm{C}$, pero en algunas ocasiones registra valores bajos, de hasta $15^{\circ} \mathrm{C}$, temperaturas no ideales para la tilapia roja, que es una especie de clima cálido. Las temperaturas letales para la tilapia roja se encuentran entre los $10^{\circ} \mathrm{C}$ y los $11^{\circ} \mathrm{C}$; su alimentación cesa por debajo de los $16-17{ }^{\circ} \mathrm{C}$ y las enfermedades o muertes se producen cuando se las maneja por debajo de los $16-17^{\circ} \mathrm{C}$. La reproducción se inhibe cuando las temperaturas se sitúan por debajo de $\operatorname{los} 20^{\circ} \mathrm{C}$. Para su crecimiento se necesita entre $29^{\circ} \mathrm{C}$ y 31 ${ }^{\circ} \mathrm{C}$. Cuando los peces son alimentados a saciedad, el crecimiento se manifiesta tres veces superior que a los $20-22^{\circ} \mathrm{C}$. Cuando la temperatura excede los $37-38^{\circ} \mathrm{C}$ se producen también problemas por estrés [4-7].

La temperatura es el factor que determina el ritmo del crecimiento de los peces, ya que los procesos bioquímicos en la fisiología de los animales se aceleran con un aumento de ésta; los peces de aguas cálidas inician su crecimiento a una temperatura por encima de los $18{ }^{\circ} \mathrm{C}$, esto impide su cultivo en regiones montañosas. En regiones con estaciones frías, los peces crecen solamente durante el verano, mientras que los trópicos muestran siempre un crecimiento constante durante todo el año [8].

El nivel de oxígeno disuelto en un estanque de acuicultura es el parámetro más importante en la calidad del agua; si no hay buena cantidad de oxígeno disuelto, los organismo pueden ser vulnerables a enfermedades y parásitos, o morir por falta de este elemento; se ha comprobado que cuando se presentan niveles bajos de oxígeno no 
Sistema energéticamente eficiente y de bajo costo para controlar la temperatura y aumentar el oxígeno en estanques de cultivo de alevines de tilapia roja

aceptan el alimento, lo cual afecta el crecimiento [8].

Para lograr un crecimiento normal y una baja mortalidad, los valores mínimos de oxígeno disuelto se deben mantener por encima de 3.0 $\mathrm{mg} / \mathrm{l}$, valores menores reducen el crecimiento e incrementan la mortandad [8]; niveles superiores son deseables.

\section{Diseño DEL SISTEMA}

En el diseño del sistema se debió hacer una minuciosa selección y dimensionamiento de los equipos por utilizar, principalmente el PLC, los colectores solares y la motobomba; el resto de materiales son considerados insumos de fácil selección.

\section{A. Controlador lógico programable}

El equipo central de todo el sistema de automatización es un controlador lógico programable o PLC; para su dimensionamiento se definieron los siguientes criterios:

- Que sea de bajo costo, de modo que su implementación en granjas de piscicultura sea económicamente viable.

- Debe ser robusto y confiable, que ya haya tenido validación a nivel nacional.

- Su programación debe realizarse bajo software libre, evitando la adquisición de licencias que incrementarían su costo.

- Se adquirirá una pantalla para realizar una interfaz hombre-máquina (IHM), o bien el PLC debe contar con una integrada.

- Debe contener por lo menos 2 entradas analógicas compatibles con termocuplas, para la detección de la temperatura en el estanque en 2 puntos diferentes.
- Debe permitir el almacenamiento de variables (muestreo) para hacer la validación del sistema.

- Debe contar con cable de comunicación entre el PLC y el computador de bajo costo o construcción simple.

- Debe contar con funciones de fecha y hora, además de su almacenamiento por mínimo 6 horas.

- Preferiblemente, debe contar con software simulador para realizar las pruebas previas a la puesta en marcha del sistema (opcional).

Tras un sondeo entre diferentes fabricantes, se optó por un PLC Unitronics M91-2-UN2, porque cumple con la mayoría de las condiciones descritas:

- Su costo es de, aproximadamente, $\$ 950.000$,oo.

- Ha tenido aplicaciones a nivel local y nacional con excelentes resultados en el campo de la automatización industrial.

- El software de programación se llama "U90 Ladder", de descarga gratuita y libre uso desde la página del fabricante.

- Tiene una IHM integrada, monocromática de 2 líneas, con limitaciones pero suficiente para hacer una IHM que muestre estados del proceso.

- Tiene 2 entradas analógicas configurables para diferentes tipos de señales, voltaje, corriente, resistencia, etc., entre ellas escalización directa para diferentes tipos de termocuplas.

- Contiene una función de direccionamiento indirecto que permite hacer un almacenamiento de hasta 256 registros en las variables llamadas "MI" tipo entero. 
- El cable de comunicación es entregado sin costo adicional al adquirir el PLC; además, es de fácil construcción en caso de requerirse un reemplazo.

- Cuenta con funciones de fecha y hora almacenables por batería interna hasta por 7 días.

- No cuenta con simulador, el algoritmo debe probarse directamente conectando el computador al PLC mediante el software U90 Ladder.

Esta última característica no es vital para el desarrollo del proyecto, puesto que se puede prescindir del simulador al contar con el PLC real y así poder probar el algoritmo de control diseñado.

\section{B. Colectores solares}

En la consulta realizada sobre colectores solares se encontraron diferentes tipos, ellos son:

1) Colector de placa plana con cubierta: Es el más usado para calentar el agua en los hogares y para el sistema de calefacción. Un colector de placa plana de cubierta se compone básicamente de una caja metálica con aislamiento, con una cubierta de vidrio o de plástico y con una placa oscura que absorbe calor. La radiación solar es absorbida por la placa que está construida de un material que transfiere rápidamente el calor a un fluido que circula a través de tubos en el colector. Además, la cubierta transparente evita que el viento y las brisas se lleven el calor colectado (convección). Este colector ha demostrado una muy buena relación precio/calidad y tiene una amplia gama de posibilidades para su montaje [9]. Son eficientes, pero su costo es elevado, debido a su cantidad de componentes.

2) Colectores de placa plana sin cubierta. Este tipo de colectores, sencillos y de bajo costo, consisten en un absorbedor, pero carecen de la cubierta transparente y no incluyen ningún aislamiento adicional, de manera que la ganancia de temperatura queda limitada a unos $20^{\circ} \mathrm{C}$ sobre la del aire del ambiente; son los más adecuados para aplicaciones de baja temperatura, ya que se mantienen en una temperatura de 10 y $40{ }^{\circ} \mathrm{C}$; son utilizados para la calefacción de piscinas al aire libre, pero existen otros mercados, incluidos los de calefacción de temporada en las piscinas cubiertas, calefacción de agua para lavar carros [9], y calefacción del agua utilizada en piscicultura.

Los absorbedores de estos colectores son generalmente de plástico negro tratado para resistir la luz ultravioleta, o están construidos por tubos de metal o plástico recubiertos de pigmentos ennegrecidos, por los que circula el agua (Fig. 1). Dado que estos colectores no tienen cubierta, su construcción es de muy bajo costo, pero gran parte de la energía solar absorbida se pierde principalmente por convección.

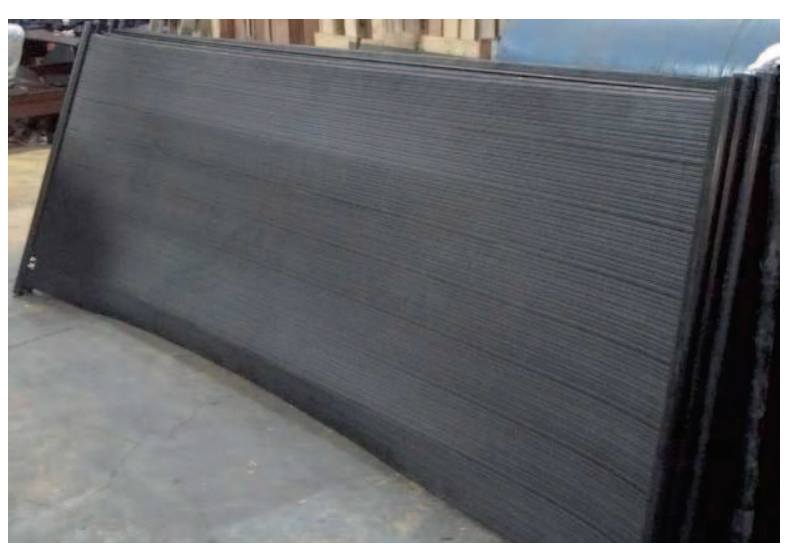

Fig. 1. Colector de placa plana sin cubierta

3) Colectores CPC estacionarios: Tienen un sistema de concentración de radiación solar tipo Concentradores Parabólicos Compuestos (CPC), para obtener temperaturas más elevadas y un mayor rendimiento; estas características se deben a que el área de pérdidas es menor al área de colección, logrando una minimización de las pérdidas y alcanzando un rendimiento cercano al $50 \%$. Son colectores concentradores, de alto rendimiento y alta calidad, de patente portuguesa [9]. 
Sistema energéticamente eficiente y de bajo costo para controlar la temperatura y aumentar el oxígeno en estanques de cultivo de alevines de tilapia roja

4) Colectores de tubos de vacío: Se componen de un conjunto de tubos de vacío, cada uno de los cuales contiene un absorbedor (generalmente una plancha de metal con tratamiento selectivo o de color negro), el cual recoge la energía solar y la transfiere a un fluido portador (caloportador). Gracias a las propiedades aislantes del vacío, las pérdidas de calor son reducidas [9] y pueden alcanzarse temperaturas en el rango de $77{ }^{\circ} \mathrm{C}$ a $177{ }^{\circ} \mathrm{C}$. De esta manera, este tipo de colectores resultan particularmente apropiados para aplicaciones de alta temperatura. Son hasta unos 30\% más eficientes que los colectores planos, pero son bastante costosos, por unidad de superficie suelen costar aproximadamente el doble que un colector de placa plana [9].

Para este diseño se trabajó con colectores de placa plana sin cubierta, porque, aunque reducen su eficiencia al perder calor por convección, su costo es más bajo. El rango de temperaturas en que opera resulta ser adecuado para los alevines de tilapia roja, debido a que la temperatura deseada es de $28^{\circ} \mathrm{C}$.

Una vez seleccionado el tipo de colector solar, se cotiza con un proveedor local, que ofrece unos construidos en polipropileno, capaces de captar. Con este dato se puede calcular el área total que deben tener, dependiendo de la cantidad de agua que se desea calentar, así:

Volumen de agua:

$$
v=3 m * 3 m * 0.6 m=5.4 m^{3}(1)
$$

Densidad del agua:

$$
\rho=1000 \mathrm{~kg} / \mathrm{m}^{3}
$$

Masa de agua por calentar:

$$
\begin{gathered}
m=\rho * v=5.4 m^{3} * 1000 \frac{\mathrm{kg}}{\mathrm{m}^{3}}(3) \\
m=5400 \mathrm{~kg}(4)
\end{gathered}
$$

Calor específico del agua:

$$
C=4.18 \frac{k j}{k g *{ }^{\circ} \mathrm{C}}(5)
$$

Temperatura final deseada:

$$
t_{f}=29^{\circ} \mathrm{C}(6)
$$

Temperatura inicial que se tiene:

$$
t_{i}=23^{\circ} \mathrm{C}(7)
$$

La demanda energética es:

$$
\begin{gathered}
E=m * C *\left(t_{f}-t_{i}\right)(8) \\
E=5400 \mathrm{~kg} * 4.18 \frac{\mathrm{kj}}{\mathrm{kg} *{ }^{\circ} \mathrm{c}} *\left(29{ }^{\circ} \mathrm{C}-23{ }^{\circ} \mathrm{C}\right)(9) \\
E=135432 \mathrm{kj}(10)
\end{gathered}
$$

El área total se calcula dividiendo la demanda energética por la energía del colector por metro cuadrado, valor suministrado en la ficha técnica, es:

$$
\begin{gathered}
A_{\text {Colec }}=\frac{(135432 k j)}{\left(11300 \frac{k j}{m^{2}}\right)}(11) \\
A_{\text {Colec }}=11.9851 \approx 12 \mathrm{~m}^{2}(12)
\end{gathered}
$$

El fabricante anteriormente consultado ofrece colectores de forma modular, es decir, deben adquirirse colectores de menor área de forma independiente y luego acoplarse. Cada módulo tiene de ancho por de largo, teniendo así cada uno un área de; por lo tanto, se concluye que se requieren 4 colectores solares.

Debidoalpresupuestodelproyecto,únicamentefue posible adquirir 3 de los 4 colectores proyectados, lo cual, finalmente, demandará mayor tiempo para lograr la temperatura ideal. Adicionalmente, el fabricante sugirió que los colectores se instalaran lo más cerca posible del estanque de alevines, para aumentar la eficiencia, pero no fue posible por no haber un área lo suficientemente amplia alrededor del invernadero; tampoco fue posible ubicarlo en el techo, debido al constante cambio 
de cubierta al que se ve sometido (cada 6 meses, aproximadamente), sumándose a esto la débil estructura del invernadero; por lo cual se debió ubicar un lugar adecuado para su instalación.

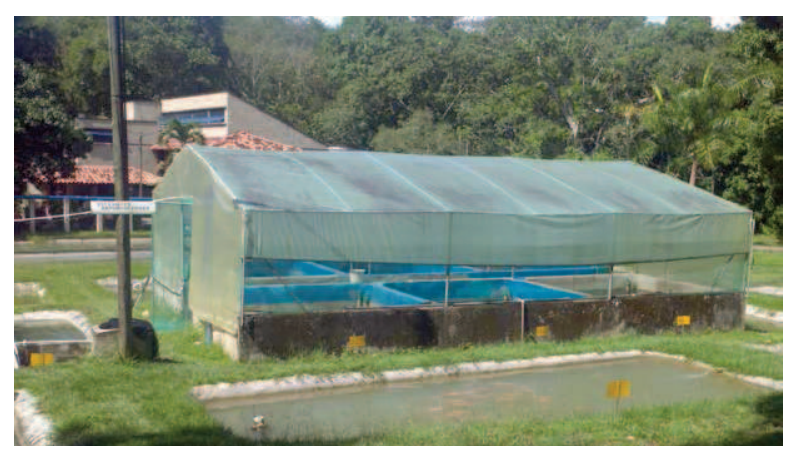

Fig. 2. Invernadero de alevines de la granja

\section{Otros elementos}

Otros elementos que se utilizaron para la implementación del sistema fueron una motobomba de $0.5 \mathrm{HP}$, con un caudal de $40 \mathrm{l} /$ min y 4 electroválvulas controladas por relé de 24 VDC, para activación automática de circuitos de agua caliente y fría, recirculación de colectores y apagado del sistema.

\section{Sistema de control}

La construcción del sistema se hace siguiendo el plano de instrumentación diseñado bajo la norma ISA S5 [10] (Fig. 3), en el cual se usaron dos termocuplas tipo J para el control de temperatura, y un flotador para el nivel, los cuales son señales de entrada al PLC.

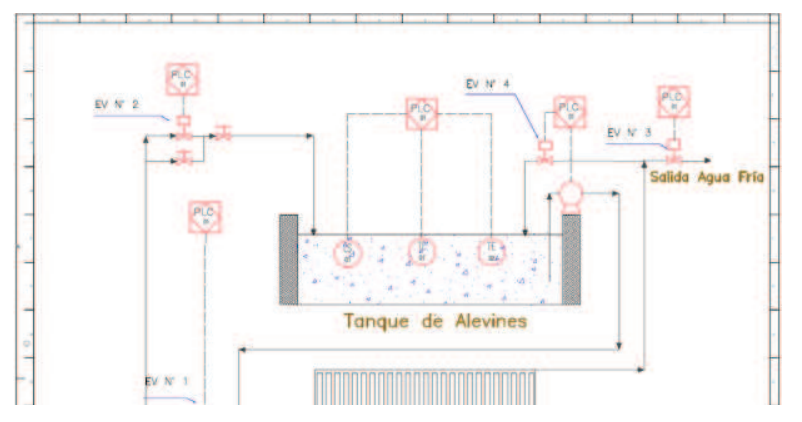

Fig. 3. Plano de instrumentación
El sistema de control está centralizado en el PLC, y está a cargo de recibir la información del proceso a través de las 2 termocuplas y el flotador de nivel; de acuerdo con el algoritmo programado, encenderá o apagará las electroválvulas $(\mathrm{EV})$ y la motobomba, así (Fig. 4):

- Si el tanque está con nivel bajo se activan EV1 y EV2, con el fin de suministrar agua al estanque.

- Si el tanque está lleno, pero la temperatura está baja, se activa la motobomba para extraer agua del estanque, haciéndola pasar por los colectores solares y regresándola al estanque a través de la EV4.

- Si la temperatura ya está lo suficientemente alta, entonces se activan EV1 y EV3 para hacer circular agua fría por los colectores y desecharla, esto con la única función de mantener agua sobre los colectores y evitar daños.

- Si la temperatura en el estanque es mucho más alta de lo deseado, entonces se debe enfriar; para esto se activa EV2, que permite el ingreso de agua fría directamente al estanque.

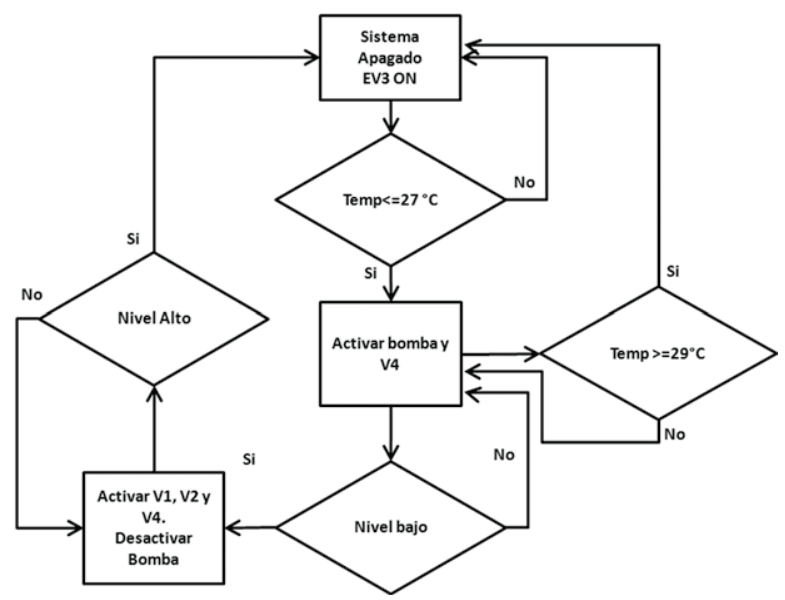

Fig. 4. Diagrama de flujo

El control con PLC requiere de todo un diseño eléctrico (Fig. 5) que contenga las protecciones, accionamientos y conexiones que permitan al PLC recibir información del proceso, ejecutar el 
Sistema energéticamente eficiente y de bajo costo para controlar la temperatura y aumentar el oxígeno en estanques de cultivo de alevines de tilapia roja

algoritmo y, a partir de allí, enviar órdenes a los actuadores.

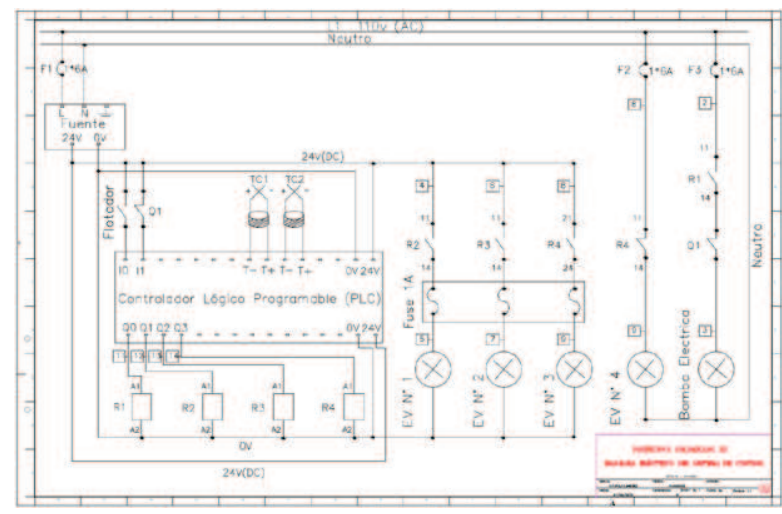

Fig. 5. Plano eléctrico del gabinete de control

1) Programa de PLC: El programa de PLC se diseñó en lenguaje ladder, que es el más universal para la programación de estos dispositivos; su sintaxis es similar a la lógica cableada, y su interfaz es gráfica, haciéndola una herramienta ideal para diseñar control lógico [11]. En este caso se utilizó un programa extenso, que no es posible incluir en su totalidad, por lo tanto, se explicarán únicamente algunas de sus líneas de código que se consideran de gran importancia.

Dado que el proyecto depende de la energía solar para poder calentar el agua, esta sección del programa hace referencia al bloque del PLC encargado de comandar el rango de tiempo en que va operar el sistema de control, garantizando que el sistema no opere durante la noche. $\mathrm{La}$ operación del sistema de control es de 7:30 am hasta 5:30 p.m. (Fig. 4).

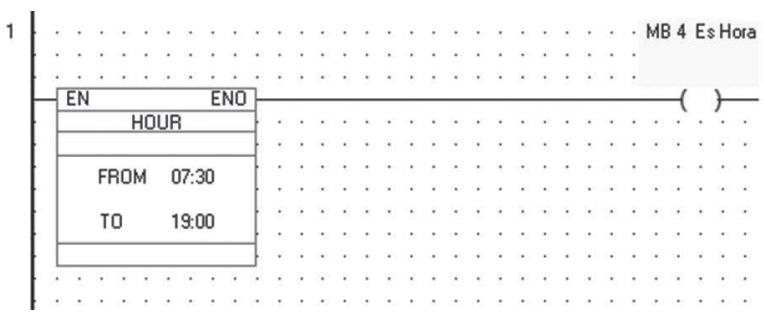

Fig. 6. Evaluación de la hora de funcionamiento

El calentamiento de agua se hace por medio de un proceso de recirculación, en el cual se enciende la motobomba para extraer agua del estanque hacia los colectores, y se enciende únicamente EV4 para permitir el ingreso de agua caliente proveniente de los colectores (Fig. 7):

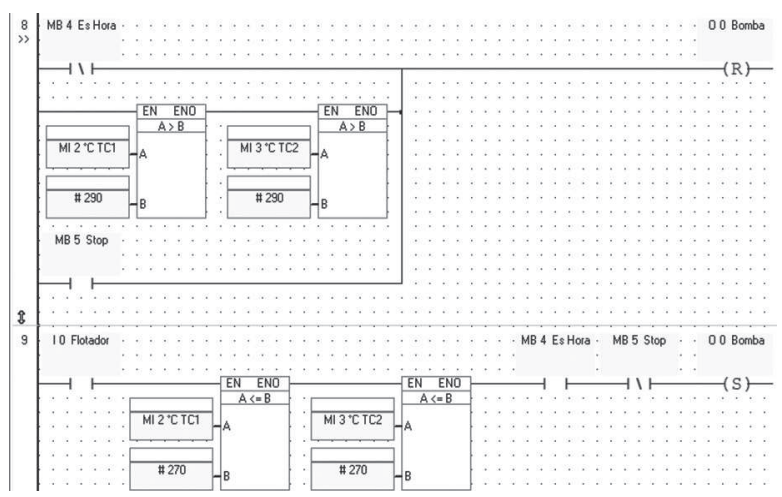

Fig. 7. Encendido y apagado de la motobomba

En caso tal de que la marca "MB 4 Es Hora" esté apagada, o la temperatura esté por encima de 29 ${ }^{\circ} \mathrm{C}$ o se dé un paro en el sistema, automáticamente la bomba deberá apagarse.

Para el encendido de la motobomba se deben cumplir varias condiciones simultáneamente: Debe haber nivel, ambas temperaturas deben estar por debajo de $27^{\circ} \mathrm{C}$, la marca "MB 4 Es Hora" debe estar encendida y no debe haber un paro en el sistema.

Otro caso que se mostrará del programa del PLC es la EV3, que se enciende cuando la temperatura está lo suficientemente elevada (Fig. 8).

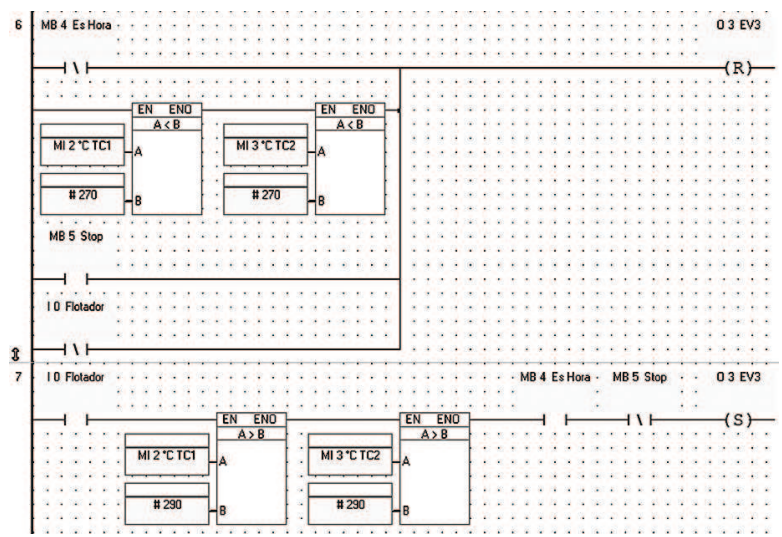

Fig. 8. Encendido y apagado de la EV3 
Analizando de forma análoga a la motobomba, el apagado se realiza bajo una combinación OR de diferentes condiciones: Si la marca "MB 4 Es Hora" está apagada, si las temperaturas están por debajo de $27^{\circ} \mathrm{C}$, si hay un paro o si no hay nivel.

El encendido se realiza bajo una combinación AND de varias condiciones: Hay nivel, ambas temperaturas son superiores a $29{ }^{\circ} \mathrm{C}$, la marca "MB 4 Es Hora" está encendida y no hay un paro.

2) Diseño de la interfaz hombre-máquina (IHM): Con la ayuda de la pantalla monocromática integrada al PLC, la cual permite a los encargados observar parámetros de interés, se elaboró una IHM; este dispositivo es muy usual en la industria, que toma como funciones el monitoreo (visualizar datos en tiempo real), las alarmas y las historias (realizar registros de las variables), entre otras.

En el PLC Unitronics la configuración por la IHM se realiza a través de displays, que presentan información utilizando las 2 líneas de la pantalla. Dado que el espacio es reducido, se debe recurrir a la creación de displays adicionales y configurar condiciones de salto automáticas o por eventos entre ellos. En el proyecto se definió que las condiciones de salto fueran por eventos, controladas por las teclas "Izquierda" y "Derecha" con forma de flechas ubicadas en la parte frontal del PLC (Fig. 9).

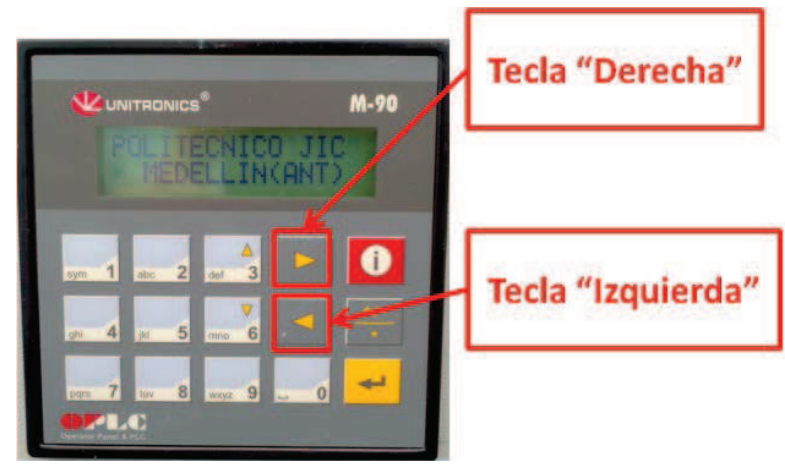

Fig. 9. Teclas de navegación de la IHM
Se diseñaron de 12 displays, así:

- Principal: Da la bienvenida al sistema

- Politécnico JIC: Se presenta información institucional (Fig. 9)

- Temperaturas: Se presenta la temperatura obtenida en ${ }^{\circ} \mathrm{C}$ por las 2 termocuplas (Fig. 10)

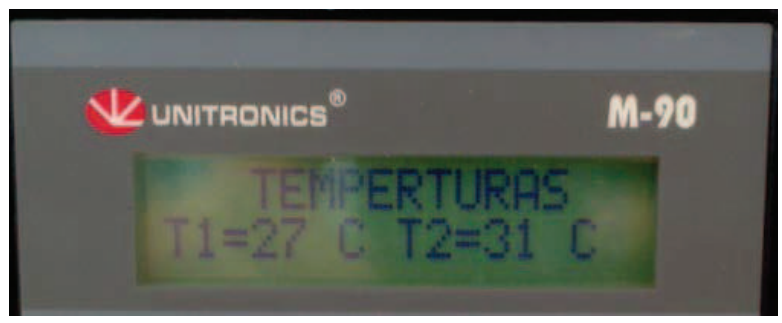

Fig. 10. Display “Temperaturas"

- Estado de nivel vs. motobomba: Se muestra el estado de nivel (alto o bajo) y de la motobomba (encendida o apagada) (Fig. 11).

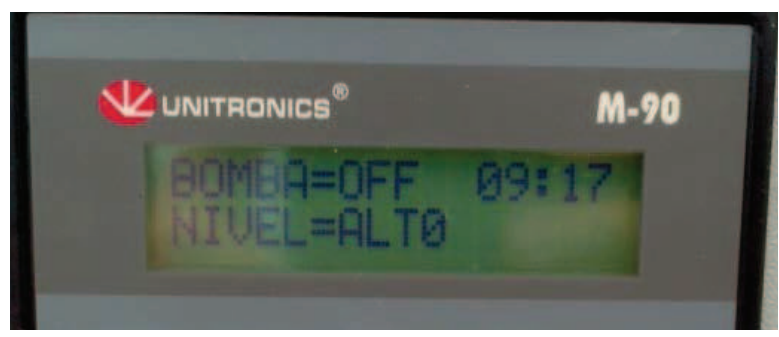

Fig. 11. Display de estados del nivel y la bomba

- Estado de las válvulas: Se muestra el estado de las 4 electroválvulas, indicando si están encendidas o apagadas (Fig. 12)

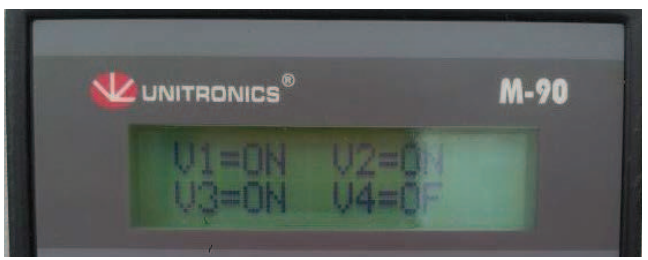

Fig. 12. Display de estado de las válvulas

- Autores: Se presentan los autores del proyecto

- Asesores: Se presentan los asesores del proyecto 
Sistema energéticamente eficiente y de bajo costo para controlar la temperatura y aumentar el oxígeno en estanques de cultivo de alevines de tilapia roja

- Sobrecarga: Indica si se ha presentado una sobrecarga en la motobomba porque se ha disparado el guardamotor.

- Paro de emergencia: Indica si se ha presionado un paro de emergencia (Fig. 13).

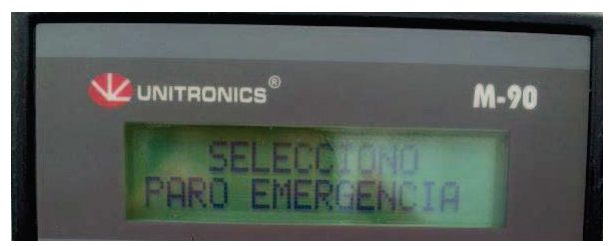

Fig. 13. Display "Paro de emergencia"

- Calentando agua: Indica si el sistema se encuentra en proceso de calentamiento de agua.

- Temperatura ideal: Indica que el sistema ha alcanzado la temperatura ideal.

-Almacenamiento de temperatura: Indica que se ha iniciado el proceso de muestreo de la temperatura cada hora (únicamente para validación).

- Paro de almacenar temperatura: Indica que se ha detenido el muestreo de temperatura.

\section{IMPLEMENTACIÓN DEL SISTEMA}

La implementación de este sistema automático tiene diferentes etapas, tanto de instalación mecánica como eléctrica, teniendo por delante los retos de acoplarse perfectamente a un sistema existente y en funcionamiento desde años atrás. Para esta instalación se requiere vaciar el tanque durante el tiempo que ella dura, para primero realizar la implementación, las pruebas y la puesta en marcha, y luego poder ingresar agua y finalmente alevines, cuando el sistema se encuentre operando de forma autónoma y segura.

\section{A. Instalación del PLC}

Para proteger e instalar adecuadamente el PLC se diseña un gabinete de control, que es construido siguiendo fielmente el plano eléctrico (Fig. 5).
Dentro del gabinete se instala la parte eléctrica: la fuente de voltaje (para conversión de 110 AC a 24 DC), 1 guardamotor y 4 relés (Fig. 14).

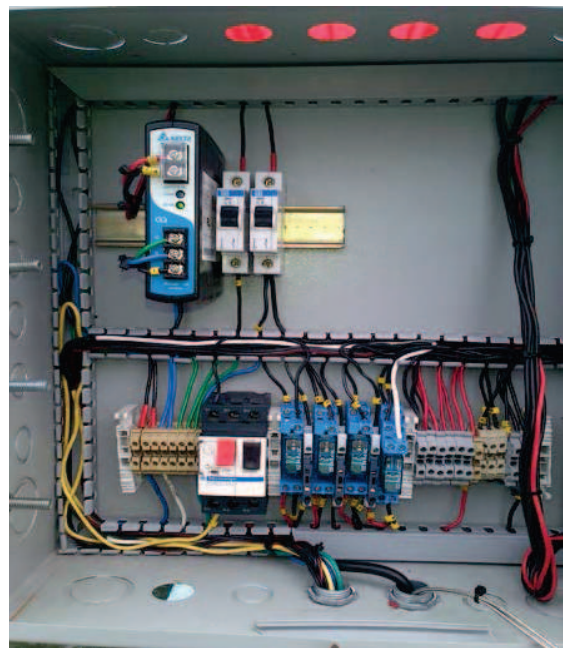

Fig. 14. Gabinete de control

\section{B. Instalación de las termocuplas}

Las dos termocuplas se implementaron en lugares diferentes del estanque, para ampliar el área de la toma de mediciones, en el extremo opuesto de la entrada de agua caliente al estanque (Fig. 15).

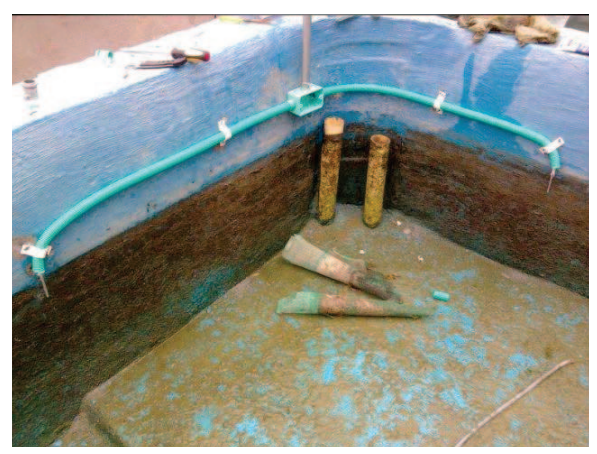

Fig. 15. Instalación termocuplas tipo J

\section{Instalación del flotador}

Los estanques no requieren control de nivel por exceso de agua, puesto que tienen ya implementado un sistema de rebose; sin embargo, el sistema diseñado contiene una motobomba para la extracción de agua del estanque. Un mal funcionamiento del sistema podría generar un 
vaciado no deseado del tanque, perjudicando los alevines, o un encendido de la motobomba en vacío, ocasionando daños en sus sellos. Se instala un sensor de nivel por flotador que envíe una señal al PLC, para que sea verificado el nivel adecuado antes de encender la motobomba (Fig. 16).

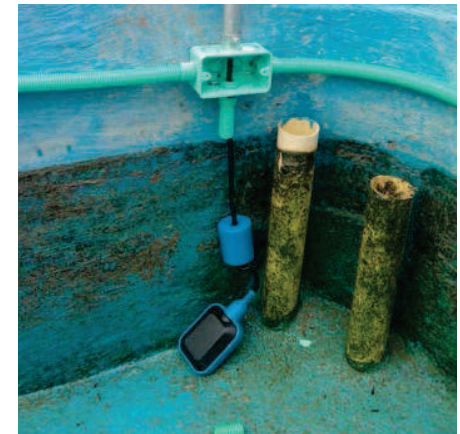

FIG. 16. Flotador para control de nivel

\section{Instalación de la motobomba y de las electroválvulas}

La motobomba instalada tiene las siguientes características técnicas:

$\begin{array}{ll}\text { Marca: } & \text { Pedrollo } \\ \text { Referencia: } & \text { PKm60 } \\ \text { Potencia: } & 0.5 \mathrm{hp} / 0.37 \mathrm{~kW} \\ \text { Alimentación: } & 110 \mathrm{VAC} 60 \mathrm{~Hz} \\ \text { Velocidad nominal: } & 3450 \mathrm{rpm} \\ \text { Caudal: } & 40 \mathrm{l} / \mathrm{min} .\end{array}$

Altura máxima de succión: $8 \mathrm{~m}$

Dimensiones: 243x152x120 mm (Largo x Ancho $\mathrm{x}$ Alto)

Diámetro de descarga: 1"

Diámetro entrada de succión: 1"

Las bombas Pedrollo PKm60 tienen un motor de eficiencia mejorada, con una temperatura de operación de $20{ }^{\circ} \mathrm{C}$ por debajo de otros, lo que le permite tener menor consumo energético, ser más silencioso y aumentar su vida útil [12]. Para aumentar la eficiencia de los motores eléctricos es común utilizar arrancadores suaves y variadores de velocidad. Los arrancadores suaves se recomiendan en aplicaciones donde no se regula velocidad, pero se está haciendo un frecuente arranque de los motores, lo que genera un alto consumo energético por el sobrepico de corriente para vencer la inercia inicial cero. Los variadores se usan en el caso de que se requiera regular la velocidad, también pueden servir como arrancadores suaves.

En el caso de este proyecto existe la prioridad de mantener bajos los costos de instalación, de modo que sean más asequibles para todo tipo de cultivos y población; por ello no se recomienda la utilización de variadores, pues no se regula velocidad en ningún momento, y la utilización de arrancadores suaves es opcional, puesto que el sistema únicamente encenderá una vez en la mañana, para subir la temperatura que se perdió durante la noche, y ocasionalmente durante el día, en caso de que la temperatura baje por factores ambientales. El arranque y paro se genera por contactor.

La motobomba se instala en un extremo del estanque, utilizando uniones universales para su fácil desmontaje; además, se instala un filtro para su protección. Como la motobomba tiene la entrada de agua directamente del estanque, lleva una malla con aperturas inferiores a $1 \mathrm{~mm}$, para garantizar que no succionará alevines en el proceso.

Las electroválvulas se conectan con tubería PVC; además, se instalan válvulas manuales by-pass para operar el sistema de forma manual en caso de presentarse fallas eléctricas o en el sistema de control. El estado normal de estas válvulas manuales durante la operación debe ser cerrado.

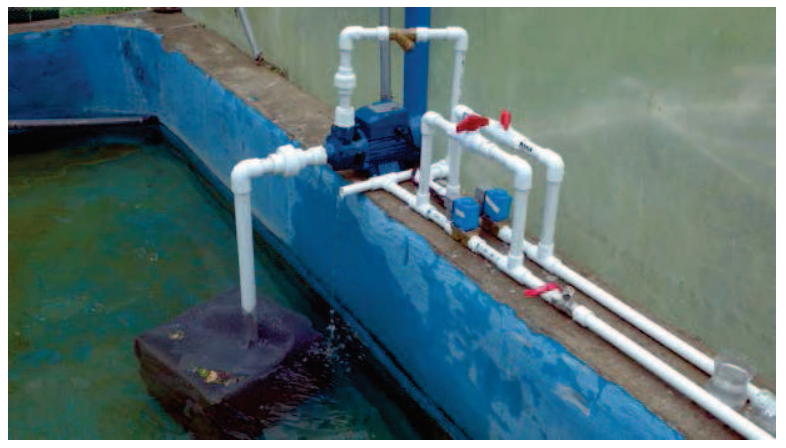

Fig. 17. Instalación de la motobomba y las electroválvulas 
Sistema energéticamente eficiente y de bajo costo para controlar la temperatura y aumentar el oxígeno en estanques de cultivo de alevines de tilapia roja

\section{E. Instalación de los colectores solares}

Los colectores solares deben ubicarse en un lugar que tenga la mayor exposición a energía solar posible; sobre el techo del invernadero es ideal, pero, como ya se anotó, esto no fue posible debido a la débil estructura del invernadero y a que la cubierta es cambiada cada 6 meses, aproximadamente. Otra alternativa es ubicarlos sobre una superficie, de manera inclinada, con una pendiente del $15 \%$, lo más cerca posible al invernadero para no perder eficiencia; esto tampoco fue posible, puesto que alrededor del invernadero se encuentra gran cantidad de piscinas de reproducción (Fig. 2).

Se ubicó una zona con un área libre a $15 \mathrm{~m}$ del invernadero, a la misma altura del estanque, evitando que se generen gastos energéticos y pérdida de caudal si se tuviera que vencer una presión hidrostática.

Se hicieron mediciones de temperatura a la salida del colector y se compararon con las de la llegada de agua al estanque, obteniéndose la misma temperatura con una precisión de \pm 0.1 ${ }^{\circ} \mathrm{C}$; por lo tanto, la distancia no generó pérdidas significativas en esta variable. Esto debido a que la tubería también está expuesta a la radiación solar y, además, el PVC tiene un coeficiente de conductividad térmica bajo, de 0.12 a 0.25 [13], comparado con los metales que tienen un coeficiente alto, por ejemplo, el acero $(45$, el aluminio (200 y el oro (308.2.

Dado que la ubicación no afectó la eficiencia y que el terreno tiene una inclinación natural que cumple con los requisitos dados por el fabricante (Fig. 18), se instalaron definitivamente los colectores en este espacio.

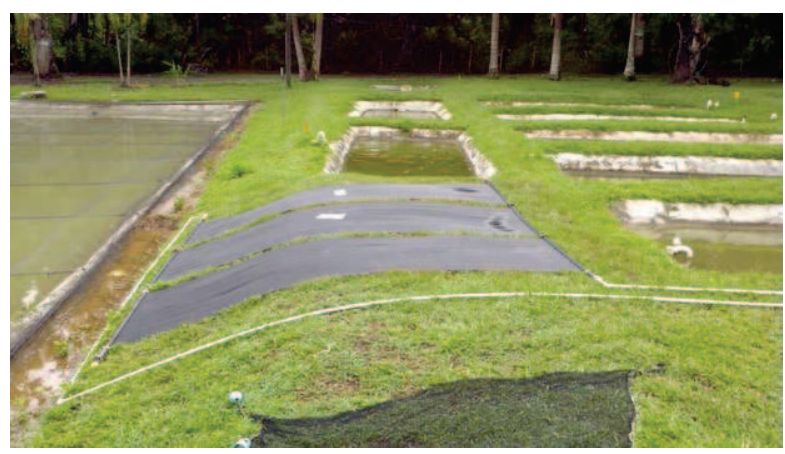

Fig. 18. Instalación de los colectores solares

Se instala una tubería de ingreso de agua fría por la parte inferior, y una de salida por la parte superior, de modo que se cumpla con el principio físico del termosifón.

\section{F. Instalación del sistema de aumento de oxígeno}

El costo de instalar un oxímetro por estanque haría que el valor total del montaje se duplique, haciendo económicamente inviable este tipo de implementación en una granja tradicional. Como sin oxímetro es imposible hacer un control en lazo cerrado (realimentado) de esta variable, se sugiere, como alternativa, implementar un sistema no controlado que contribuya al aumento de oxígeno a través del ingreso permanente de burbujas de aire en la parte inferior del estanque (Fig. 19) y evaluar si se alcanzaron los niveles adecuados, a pesar de no ser una variable controlada por realimentación.

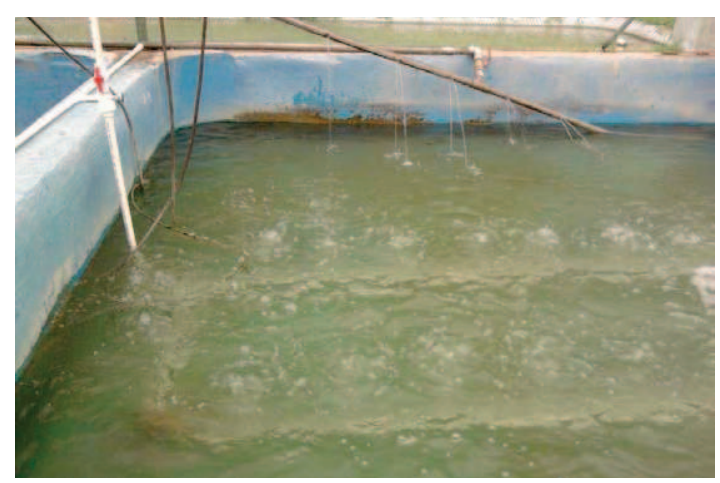

Fig. 19. Sistema de aumento de oxígeno 


\section{Resultados}

Para validar el sistema de control se hace una prueba inicial en lazo abierto para evaluar la eficiencia de los colectores solares; en esta prueba no se hace recirculación de agua, lo cual afectará la eficiencia. Se ingresa agua de la quebrada Guaracú directamente a los colectores y se envía al estanque. La prueba se realizó durante una semana, graficando en el eje horizontal el tiempo en horas, y en el eje vertical, en línea continua, la temperatura en ${ }^{\circ} \mathrm{C}$, y en línea discontinua la hora del día.

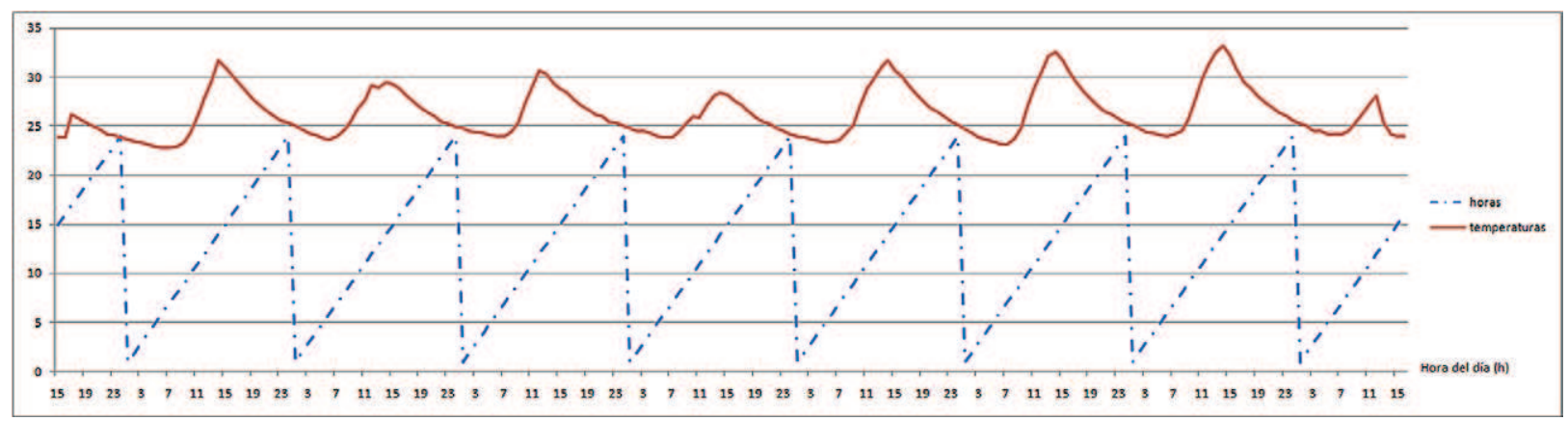

Fig. 20. Validación de temperatura en lazo abierto

En la Fig. 20 se observa una temperatura variable de forma cíclica con los días. Aproximadamente a las 7 a.m. inicia el calentamiento del agua, el cual tiene tendencia al aumento hasta las 3 ó 4 p.m. En algunos días se alcanzan temperaturas superiores a los $30^{\circ} \mathrm{C}$. A partir de esta hora, el Sol se oculta en una montaña al occidente y no llega su energía a los colectores, por lo cual empieza el enfriamiento, que es constante hasta las 7 a.m., cuando empieza nuevamente el calentamiento.

Durante las noches se recomienda usar cobijas térmicas que disminuyan la pérdida de temperatura en el agua, o implementar un sistema con resistencias eléctricas que realice el calentamiento ante la ausencia de energía solar.

Nótese que se tenían tres condiciones en contra de la eficiencia de los colectores: la distancia entre el estanque y los colectores, la cantidad de colectores (se calcularon cuatro, pero se implementaron tres) y el hecho que no se realiza recirculación. Estas tres condiciones no han impedido que el sistema llegue a la temperatura deseada de $28{ }^{\circ} \mathrm{C}$; se espera que no se requiera el sistema encendido todo el día, y que durante algunos lapsos se apague y la temperatura se conserve estable.

La siguiente prueba, y la final, es el sistema en lazo cerrado; para ello se pone en marcha el sistema y se deja trabajando durante varios días. La toma de temperatura se realiza en dos tanques durante un día, entre las 8 a.m. y las 6 p.m.

El tanque intervenido con el proyecto se denomina tanque 1 (TK1), y se elige como testigo otro tanque tradicional, sin control de temperatura ni sistema de aumento de oxígeno (TK2). Al principio de la toma de muestras (a las 8 a.m.) se registran temperaturas similares, pero a partir de allí se empieza a notar una pendiente más alta en el incremento de temperatura de TK1, la cual alcanza a subir hasta $30^{\circ} \mathrm{C}$ a las 4 p.m; esto debido a que el sistema se apaga a los $29{ }^{\circ} \mathrm{C}$ y la temperatura tiene un comportamiento inercial. Durante el resto del día se mantiene alrededor de $\operatorname{los} 28^{\circ} \mathrm{C}$ deseados. 
Sistema energéticamente eficiente y de bajo costo para controlar la temperatura y aumentar el oxígeno en estanques de cultivo de alevines de tilapia roja

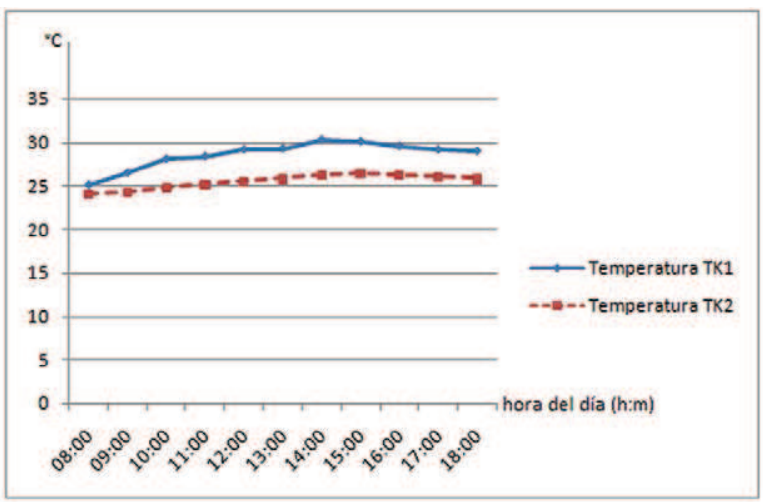

Fig. 21. Validación de temperatura en lazo cerrado

En la prueba final también se tomaron muestras de la variable oxígeno con la misma periodicidad (Fig. 22). En este caso se hizo con un oxímetro externo, no conectado al PLC, solo con fines de validación.

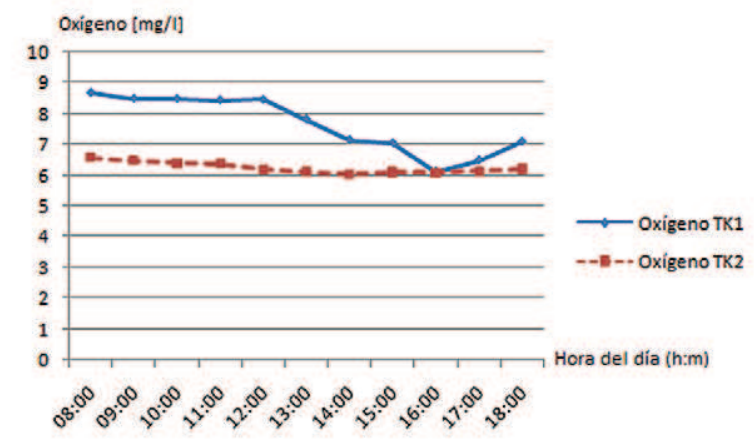

Fig. 22. Validación del aumento de oxígeno

Desde el inicio del muestreo, en el TK1 se obtuvo un nivel superior de oxígeno que en el TK2, esto porque el sistema no tiene control y siempre está encendido, por lo cual durante la noche no tuvo valores bajos. Aproximadamente a las 4 p.m., el TK1 tuvo una caída en sus valores de oxígeno, llegando aproximadamente a $6 \mathrm{mg} / \mathrm{l}$, valor superior al mínimo permitido para los alevines, de $5 \mathrm{mg} / 1$.

\section{Conclusiones}

En el cultivo de tilapia roja es crítico mantener la temperatura alrededor de los $28^{\circ} \mathrm{C}$, con el fin de hacer más eficiente su metabolismo y, así, su crecimiento; especialmente, se debe controlar en la etapa de crecimiento de los alevines, debido a su vulnerabilidad.

La automatización con PLC de este cultivo, donde se controlan variables como temperatura y nivel de oxígeno, genera un proceso de producción más autónomo, seguro y eficiente.

La implementación de colectores solares de bajo costo hace que el proyecto sea económicamente viable y energéticamente más eficiente para pequeñas y medianas granjas, debido a su baja inversión inicial y el ahorro energético ocasionado por la utilización de la energía alternativa.

Durante la noche se pueden presentar valores bajos de temperatura, cuyo impacto negativo se puede disminuir con la utilización de cobijas térmicas o sistemas eléctricos de calentamiento, aunque estos acarrearán costos adicionales de servicios públicos.

\section{Agradecimientos}

Al Politécnico Colombiano Jaime Isaza Cadavid, por la financiación del proyecto "Diseño e implementación de un sistema que permita mantener o controlar parámetros importantes para la supervivencia y adecuado crecimiento de los alevines de tilapia roja", con centro de costos 2061080233. Además, al profesor Orlando Palacio, por su asesoría en temas de transferencia de calor, y a las profesoras Lucy Arboleda y Mónica Taborda, por su asesoría en temas de piscicultura.

\section{REFERENCIAS}

[1] Polyculture of Tilapia with marine shrimp. Green, Bartholomew. Alabama, USA: Acta del Primer Simposio Centroamericano sobre Cultivo de Tilapia. Department of Fisheries and Allied Aquacultures., 1995. 117.127. 
[2] Evaluación del crecimiento de alevines de tres especies de tilapia (Oreochromis sp.) en aguas duras, en la región de la Cañada, Oaxaca, México. Oaxaca, México: Revista AquaTIC, N. ${ }^{\circ} 20,2004$, pp. 38-43.

[3] D. Usgame, G. Usgame and C. Valverde, Agenda productiva de investigación y desarrollo tecnológico para la cadena productiva de la Tilapia. Bogotá: Ministerio de Agricultura, Colombia, 2007.

[4] Ministerio de Agricultura de Argentina. Acerca del cultivo de la tilapia nilotica y tilapia roja. [Online]. [Citado: 12/16/2013]. http://www.minagri.gob.ar/SAGPyA/ pesca/acuicultura/01=Cultivos/01Especies/_archivos/ 000008 Tilapia/071201_Acerca\%20de1\%20 Cultivo\%20de\%20Tilapia\%20Roja\%20 o\%20Del $\% 20$ Nilo.pdf.

[5] W. Berrío, Parámetros importantes en la cría de alevines de tilipia roja. [interv.] Rubén Vásquez. Marzo 22, 2013.

[6] J. Dávila, Crecimiento de Oreochromis spp. tilapia roja cultivada en agua de mar a diferentes temperaturas. Callao: Universidad Nacional del Callao, 2008.

[7] F. Borja, L. González and V. Quintero, Diseño modelo de estanques climatizados para el cultivo de tilapia roja localizados en la zona fría del Valle del Cauca, Colombia. Palmira: Universidad Nacional de Colombia, 2003.

[8] F. Martínez, Parámetros importantes a controlar en un sistema de cultivo de peces. Santiago de Cali: s.n., 2008.

[9] C. Placco, L. Saravia and C. Cadena. Colectores solares para agua caliente. s.l.: Inenco, 2010.

[10] ISA. American National Standard ANSI/ ISA S5. s.1.: ISA, 1984.

[11] R. Vásquez, Control Lógico Programable. Medellín: Fondo Editorial ITM ISBN 978958-8351-95-7, 2010.

[12] Cater Spares. Pedrolo OEM Booster. [Online] Cater Spares, 2014. [Cited: 02 14, 2014.] http://caterspares.com/Catalogue/ Washing/Wash-Rinse-Booster-Pumps/ Pedrollo-Manufactured-Pumps/PedrolloOEM-Booster-Pump-05-HP-1BSPF40Lmin-max-60C-ModPKm60-230vAC--Monophase.

[13] Plastibages Industrial. Tabla con las características técnicas y propiedades del PVC. [Online] Plastibages Industrial, 2014. [Cited: 02 12, 2014.] http://www. plasticbages.com/caracteristicaspvc.html. 